\title{
Cascada de atención de la tuberculosis para la población indígena en Colombia: una investigación operativa
}

\author{
Jhon Edwin Polanco-Pasaje ${ }^{1}$, lader Rodríguez-Márquez¹, Kelly Yoana Tello-Hoyos², Pilar Torres- \\ Pereda ${ }^{3}$, Bertha Leonor Guzmán-Salazar ${ }^{2}$, Freddy Pérez ${ }^{4}$
}

Forma de citar

Polanco-Pasaje JE, Rodríguez-Márquez I, Tello-Hoyos KY, Torres-Pereda P, Guzmán-Salazar BL, Pérez F. Cascada de atención de la tuberculosis para la población indígena en Colombia. Rev Panam Salud Publica. 202044:e150. https://doi.org/10.26633/ RPSP.2020.150

RESUMEN

Objetivo. Construir y evaluar la cascada de atención de la tuberculosis pulmonar en la población indígena del departamento del Cauca (Colombia) e identificar las brechas existentes.

Métodos. Metodología mixta con diseño secuencial explicativo. En la primera fase se evaluó la cascada de atención de la tuberculosis pulmonar para la población indígena del Cauca. Se obtuvieron datos de fuentes secundarias y se incluyeron todos los casos diagnosticados entre el 1 de enero del 2016 y el 31 de diciembre de 2017. En la segunda fase, se aplicaron entrevistas semiestructuradas a nueve coordinadores de programa y 11 auxiliares de enfermería para explicar las brechas identificadas. Se estimaron los valores absolutos y porcentuales en cada uno de los pasos y las brechas de la cascada de atención. Se triangularon los resultados cuantitativos y cualitativos.

Resultados. Durante 2016 y 2017 se estimaron 202 sintomáticos respiratorios esperados positivos y se notificaron 106 casos de tuberculosis pulmonar en la población indígena del departamento del Cauca. Se encontró una brecha de 47,5\% para el diagnóstico, ya que solo 52,5\% de los sujetos recibieron el diagnóstico en los servicios de salud. Las explicaciones a esta brecha fueron la mala calidad de muestras y fallas en la técnica del extendido, fallas en la correcta identificación del sintomático respiratorio, acceso limitado a métodos diagnósticos como cultivo y pruebas moleculares, así como capacitación escasa y rotación alta de personal al interior de las instituciones prestadoras de servicios de salud.

Conclusiones. Las acciones del programa de control de tuberculosis deben enfocarse en reducir la brecha de detección de casos en la población indígena.

Palabras clave Tuberculosis; población indígena; servicios de salud del indígena; calidad, acceso y evaluación de la atención de salud; Colombia.

La tuberculosis $(\mathrm{TB})$ representa la principal causa de muerte por un agente infeccioso en el mundo y la incidencia estimada para el 2018 fue de 10 millones de personas (1). La mayor carga de la enfermedad se presenta en poblaciones vulnerables, entre ellas, los indígenas. Se estima una población de aproximadamente 370 millones de indígenas en el mundo, que representan menos de $5 \%$ de la población mundial; sin embargo, no se conoce la carga y el estado actual de la TB con precisión en

\footnotetext{
1 Grupo de Investigación Epidemiología, Facultad Nacional de Salud Pública, Universidad de Antioquia, Medellín, Colombia. $\triangle$ Jhon Edwin PolancoPasaje, jhon.polanco@udea.edu.co

2 Secretaría de Salud Departamental, Gobernación del Cauca, Colombia.
}

\footnotetext{
3 Instituto Nacional de Salud Pública, Cuernavaca, México.

4 Departamento de Enfermedades Transmisibles y Determinantes Ambientales de la Salud, Organización Panamericana de la Salud, Washington D.C., Estados Unidos de América.
} 
los pueblos indígenas (2,3). En general, la región de América Latina tiene una carga alta de TB, que llega incluso a 1000 casos por 100000 indígenas, en particular en comunidades de la Amazonía brasilera (3-5).

La carga alta de TB en ciertas poblaciones podría explicarse por determinantes sociales estructurales y proximales. Una revisión sistemática reciente informó que el consumo de sustancias psicoactivas, la inseguridad alimentaria y el consumo de tabaco fueron los principales determinantes sociales proximales que contribuyeron a la TB en los pueblos indígenas (2). En América Latina y el Caribe se destaca la importancia de la coinfección por el virus de la inmunodeficiencia humana (VIH) y la TB multirresistente como los dos factores relacionados con los servicios de salud más importantes que aumentan la mortalidad en los pueblos indígenas de la Región. Además, la mejora en el saneamiento y el acceso al agua potable, ambos determinantes ambientales, permiten la reducción de morbilidad por TB en la Región (6).

En Colombia, para el año 2017 se reportaron 14480 casos de $\mathrm{TB}$, de los cuales $5,4 \%$ se presentó en indígenas. La tasa de incidencia nacional fue de 26,3 casos por 100000 habitantes (7). En el departamento del Cauca, la incidencia de TB en 2017 fue de 17,5 casos por 100000 habitantes y 32\% de los casos diagnosticados se concentraron en población indígena. Además, respecto a los casos nuevos confirmados por bacteriología, la detección fue de solo $19,3 \%$ (8). Este valor se mantiene muy lejos de la meta de detección de más de $90 \%$ de casos de TB (9).

Pese a esto, no se tiene conocimiento más amplio respecto al control de la enfermedad y se desconocen las brechas en la prestación de servicios en TB que permitan la reorientación de intervenciones para los pacientes pertenecientes a las etnias indígenas del departamento. La cascada de atención es una herramienta con pasos y brechas secuenciales sobre un proceso de atención que permite medir el rendimiento de la atención de un programa (10). Este estudio construyó y evaluó la cascada de atención de la tuberculosis pulmonar (TBP) en la población indígena del departamento del Cauca durante el período 2016-2017 e identificó las brechas existentes en la cascada a fin de contribuir con evidencia que apoye el direccionamiento de programas y políticas para la prevención y control de la enfermedad.

\section{MATERIALES Y MÉTODOS}

El estudio se realizó con metodología mixta con un diseño secuencial explicativo (11). La primera fase cuantitativa consistió en un diseño descriptivo para evaluar la cascada de atención de la TBP en la población indígena del Cauca. Con estos hallazgos, se inició una fase de investigación cualitativa donde se buscó explicar o apoyar lo encontrado en la primera fase (12).

El estudio se realizó en el departamento del Cauca, ubicado en la zona suroeste de Colombia. El Cauca es el segundo departamento con más población indígena en el país y para 2017, 20,5\% de la población se autorreconocía como indígena. En contraste, para 2018 en la población general colombiana este porcentaje era de solo $4 \%$.

La primera fase incluyó el censo de la población perteneciente a cualquier etnia indígena del Cauca registrada con TBP durante el 2016-2017. Este período permitió garantizar el seguimiento 18 meses posteriores a la terminación del tratamiento. Se excluyeron pacientes con TB extrapulmonar registrados en la base de datos del programa. En la segunda fase se aplicó un muestreo cualitativo de casos extremos entre instituciones prestadoras de servicios de salud (IPS) (13), y se tuvo en cuenta aquellos municipios con mayor y menor incidencia de TBP en la población indígena durante el período de estudio (14). Se incluyeron a coordinadores y auxiliares del programa de TB en cada institución seleccionada.

\section{Variables y definiciones}

En la primera fase se acogieron recomendaciones metodológicas propuestas por Subbaraman R. et al. para la construcción de cascadas de atención $(15,16)$. La cascada de atención es un modelo que contiene pasos (número de personas que logran un punto en la atención) y brechas (diferencia entre los pasos, que representa a las personas con resultados subóptimos) (16).

Para este estudio se definieron cinco pasos: i) sintomáticos respiratorios (SR) esperados positivos (17), ii) pacientes diagnosticados con TBP, iii) pacientes que iniciaron tratamiento, iv) pacientes que completaron tratamiento, y v) pacientes que completaron tratamiento y permanecieron vivos 18 meses después. Se establecieron cuatro brechas: i) pacientes con TBP no diagnosticados, ii) pacientes que no iniciaron tratamiento, iii) pacientes con fracaso, muerte o pérdida durante el tratamiento, $\mathrm{y}$ iv) pacientes que fallecieron durante los primeros 18 meses posteriores a la terminación del tratamiento (cuadro 1). El último paso se obtuvo por comparación de los pacientes que finalizaron tratamiento con los registros de defunciones. Para estimar el total de SR esperados positivos en la población se tuvo en cuenta el índice de positividad de la baciloscopia (BK), el cual es resultado del comportamiento histórico observado en población colombiana $(18,19)$.

Se tuvieron en cuenta las siguientes definiciones operativas: i) caso de $\mathrm{TBP}$, ii) paciente nuevo, iii) reingreso tras recaída, iv) curado, v) pérdida en el seguimiento, vi) fallecido durante el tratamiento, y vii) no evaluado (20). La población indígena se calculó según las proyecciones censales para el primer paso de la cascada y para los siguientes pasos se tuvo en cuenta el autorreconocimiento como indígena según los registros del programa de control de TB.

Para la segunda fase se aplicaron entrevistas semiestructuradas (21). Se exploraron las percepciones del personal de salud sobre las barreras para la realización de los tres ejes temáticos para el control de la enfermedad identificados a través de la revisión de literatura y lineamientos operativos del programa: búsqueda de SR, detección de casos y seguimiento al tratamiento. En el primer eje temático, las preguntas estuvieron orientadas a identificar aspectos relacionados con el contacto con los servicios de salud, planificación de la búsqueda e implementación de las estrategias planeadas. En el segundo eje se indagó sobre los métodos de detección utilizados y su acceso. Por último, en el tercer eje se rastrearon aspectos relacionados con el inicio, el seguimiento y la vigilancia del tratamiento.

\section{Recolección de información}

En la fase 1 se incluyeron todos los casos diagnosticados entre el 1 de enero de 2016 y el 31 de diciembre de 2017. Se obtuvieron datos de fuentes secundarias (base de datos del programa departamental de TB e informes reportados por las IPS) con cierre a diciembre de 2018. Los datos se validaron con otros subsistemas oficiales de información en salud de Colombia. Las 
CUADRO 1. Definiciones, métodos y fuentes de información de la cascada de atención para la tuberculosis pulmonar en la población indígena del Cauca, Colombia (2016-2017)

\begin{tabular}{|c|c|c|c|c|}
\hline Categoría & Pasos y brechas & Definición operativa & Método de estimación & Fuente de la información \\
\hline \multirow[t]{2}{*}{ Acceso a la atención } & Paso 1 & $\begin{array}{l}\text { Total de SR esperados positivos a la } \\
\text { baciloscopia en la población indígena } \\
\text { del Cauca }\end{array}$ & $\begin{array}{l}\text { Los SR esperados se estimaron con } 5 \% \text { de la } \\
\text { mitad de la población indígena mayor de } 15 \\
\text { años, según la Circular externa } 000058 \text { del } \\
2009 \text { del Ministerio de la Protección Social. } \\
\text { Luego se tuvo en cuenta el índice de } \\
\text { positividad observado de la baciloscopia para } \\
\text { la población colombiana. }\end{array}$ & $\begin{array}{l}\text { Censo poblacional, Circular externa } \\
000058 \text { del } 2009 \text { del Ministerio de la } \\
\text { Protección Social y Circular externa } \\
000055 \text { de } 2016 \text { del Ministerio de Salud y } \\
\text { Protección Social (18) }\end{array}$ \\
\hline & Brecha 1 & $\begin{array}{l}\text { Total de pacientes con TBP no } \\
\text { diagnosticados }\end{array}$ & $\begin{array}{l}\text { Se estimó como la diferencia entre el "paso 1" } \\
\text { y el "paso 2". }\end{array}$ & $\begin{array}{l}\text { Censo poblacional, Circular externa } \\
000058 \text { del } 2009 \text { del Ministerio de la } \\
\text { Protección Social, Circular externa } \\
000055 \text { de } 2016 \text { del Ministerio de Salud } \\
\text { y Protección Social (18) y base de datos } \\
\text { del programa departamental de control de } \\
\text { la TB, informes trimestrales reportados } \\
\text { por las IPS }\end{array}$ \\
\hline \multirow[t]{3}{*}{$\begin{array}{l}\text { Diagnóstico e inicio de } \\
\text { tratamiento }\end{array}$} & Paso 2 & $\begin{array}{l}\text { Total de pacientes diagnosticados } \\
\text { con TBP }\end{array}$ & $\begin{array}{l}\text { Total de pacientes notificados como casos de } \\
\text { TBP en población indígena }\end{array}$ & $\begin{array}{l}\text { Base de datos del programa } \\
\text { departamental de control de la TB, } \\
\text { informes trimestrales reportados por } \\
\text { las IPS. Se validó el tipo de pueblo } \\
\text { indígena en los pacientes registrados con } \\
\text { pertenencia étnica indígena mediante las } \\
\text { fichas de tratamiento }\end{array}$ \\
\hline & Brecha 2 & $\begin{array}{l}\text { Total de pacientes diagnosticados } \\
\text { con TBP que no iniciaron tratamiento }\end{array}$ & $\begin{array}{l}\text { Se estimó como la diferencia entre el "paso 2" } \\
\text { y el "paso 3" }\end{array}$ & $\begin{array}{l}\text { Base de datos del programa } \\
\text { departamental de control de la TB e } \\
\text { informes trimestrales reportados por las } \\
\text { IPS }\end{array}$ \\
\hline & Paso 3 & $\begin{array}{l}\text { Total de pacientes que iniciaron } \\
\text { tratamiento }\end{array}$ & $\begin{array}{l}\text { Número de pacientes notificados que } \\
\text { registraron inicio de tratamiento }\end{array}$ & $\begin{array}{l}\text { Base de datos del programa } \\
\text { departamental de control de la TB e } \\
\text { informes trimestrales reportados por las } \\
\text { IPS }\end{array}$ \\
\hline \multirow[t]{2}{*}{$\begin{array}{l}\text { Adherencia al } \\
\text { tratamiento }\end{array}$} & Brecha 3 & $\begin{array}{l}\text { Total de pacientes con fracaso del } \\
\text { tratamiento, muerte o pérdida } \\
\text { durante el seguimiento del } \\
\text { tratamiento }\end{array}$ & $\begin{array}{l}\text { Número de pacientes con fracaso del } \\
\text { tratamiento, muerte o pérdida durante el } \\
\text { seguimiento del tratamiento }\end{array}$ & $\begin{array}{l}\text { Base de datos del programa } \\
\text { departamental de control de la TB e } \\
\text { informes trimestrales reportados por las } \\
\text { IPS }\end{array}$ \\
\hline & Paso 4 & $\begin{array}{l}\text { Total de pacientes que completaron } \\
\text { tratamiento }\end{array}$ & $\begin{array}{l}\text { Número de pacientes que lograron la curación } \\
\text { o la finalización del tratamiento }\end{array}$ & $\begin{array}{l}\text { Base de datos del programa } \\
\text { departamental de control de la TB e } \\
\text { informes trimestrales reportados por las } \\
\text { IPS }\end{array}$ \\
\hline \multirow[t]{2}{*}{$\begin{array}{l}\text { Mortalidad posterior al } \\
\text { tratamiento }\end{array}$} & Brecha 4 & $\begin{array}{l}\text { Total de pacientes que fallecieron } \\
\text { durante los primeros } 18 \text { meses } \\
\text { posterior a la terminación del } \\
\text { tratamiento }\end{array}$ & $\begin{array}{l}\text { Número de pacientes que fallecieron los } \\
\text { primeros } 18 \text { meses posterior a la finalización } \\
\text { del tratamiento }\end{array}$ & Base de datos del RUAF \\
\hline & Paso 5 & $\begin{array}{l}\text { Total de pacientes que completaron } \\
\text { el tratamiento y permanecen vivos } \\
18 \text { meses después }\end{array}$ & $\begin{array}{l}\text { Se estimó como la diferencia entre el "paso 4" } \\
\text { y la "brecha 4". }\end{array}$ & $\begin{array}{l}\text { Base de datos del RUAF módulo } \\
\text { nacimientos y defunciones y base de } \\
\text { datos del programa departamental de } \\
\text { control de la TB }\end{array}$ \\
\hline
\end{tabular}

Fuente: elaboración propia.

a Cinco por ciento de la mitad de la población mayor de 15 años es un lineamiento para el manejo programático de tuberculosis en Colombia promulgado por el Ministerio de la Protección Social en la Circular Externa N. 000058 de 2009. Este lineamiento es importante para la estimación de la meta de sintomáticos respiratorios que deben ser captados cada año, tanto para las entidades territoriales, distritos y municipios. En el documento fuente no se menciona una

justificación del porqué de esta forma de estimación.
TBP, tuberculosis pulmonar; SR, sintomático respiratorio; RUAF, Registro Único de Afiliados: módulo nacimientos y defunciones.

bases de datos consultadas se fusionaron en planillas de Microsoft Excel ${ }^{\circledR}$ para facilitar los análisis.

En la segunda fase, se aplicaron entrevistas semiestructuradas a nueve coordinadores de programa y 11 auxiliares de enfermería pertenecientes a los ocho municipios seleccionados en la muestra (cuatro con incidencia alta y cuatro con incidencia baja de TBP, incluido un municipio de incidencia baja con dos IPS) (14). Se exploraron posibles explicaciones para las brechas identificadas en la primera fase. El trabajo de campo fue realizado por personal capacitado e independiente del programa de TB en la Región.

\section{Análisis de la información}

Se describieron valores absolutos y porcentuales de características sociodemográficas (sexo, edad, municipio de procedencia, pueblo indígena y régimen de afiliación), clínicas (coinfección con VIH, pruebas de susceptibilidad a fármacos realizadas y farmacorresistencia identificada) y programáticas (período de notificación, condición de ingreso y egreso del programa). Se estimaron valores absolutos y porcentuales en cada paso y brecha de la cascada de atención. Para el análisis de datos se 
utilizaron Microsoft Excel ${ }^{\circledR}$ y STATA $14^{\circledR}$ (StataCorp, College Station, TX, Estados Unidos de América).

En el componente cualitativo se realizó un análisis temático (22). Se realizó la transcripción textual de las grabaciones de audio en Microsoft Word ${ }^{\circledR}$. Luego se ingresaron a Atlas.ti versión $8^{\circledR}$ para la codificación y el análisis. Los coordinadores de

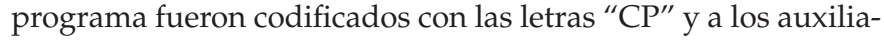
res con las letras "AP". Se asignó la letra "I" cuando pertenecía a una IPS Indígena o "E" para IPS estatal, seguido de las letras "Ai" o "Bi" según si el municipio tenía incidencia alta o baja de TB. Al final, se asignó un número correspondiente al consecutivo en el que fueron entrevistados. El análisis se desarrolló de acuerdo con las categorías antes identificadas de la cascada (captación, detección y tratamiento) y se dejó lugar para categorías emergentes (23).

La integración de métodos se logró mediante la triangulación de los resultados cuantitativos y cualitativos (12). Se realizó un ejercicio de comparación y contraste en el que, para cada brecha identificada cuantitativamente, se buscó las posibles explicaciones en los testimonios cualitativos.

El estudio fue aprobado por el Comité de Ética de la Investigación de la Facultad Nacional de Salud Pública de la Universidad de Antioquia y exonerado de revisión por parte del Comité de Revisión Ética de la Organización Panamericana de la Salud (PAHOERC).

\section{RESULTADOS}

Se estimaron 202 casos de SR esperados positivos. Sin embargo, solo 52,5\% ( $\mathrm{n}=106)$ fue diagnosticado por los servicios de salud (cuadro 2), es decir, se encontró una brecha (brecha 1) de 47,5\% de personas no diagnosticadas. Todos los pacientes diagnosticados iniciaron tratamiento, pero la brecha entre el inicio y la terminación del tratamiento (corresponde a los pacientes que iniciaron el tratamiento pero que luego fracasaron, murieron o tuvieron pérdida durante el seguimiento del tratamiento) fue de 3,5\% ( $n=7)$. Por último, $49 \%(n=99)$ de los pacientes terminaron el tratamiento y permanecieron vivos 18 meses después (figura 1 y cuadro 3).

La información proveniente del componente cualitativo no mostró diferencias sustanciales entre las percepciones de individuos con distinta profesión o provenientes de distintos municipios.

\section{Brecha 1: explicaciones frente a los casos de TBP no diagnosticados}

Mala calidad de muestras y fallas en la técnica del extendido. Los coordinadores de programa manifiestan que un número importante de muestras examinadas en el laboratorio de las IPS suelen ser de saliva. Además, como la mayoría de las muestras se extienden y fijan en campo por los auxiliares, la técnica utilizada no siempre es la mejor, lo que dificulta el proceso de tinción y lectura.

"A veces traen ese catarro bien empegotado que, si sabemos, a veces queda el bicho abajo y no se ve, es posible que esté positiva pero la capa es tan gruesa que es posible que quede oculto el bacilo, entonces ellos (los auxiliares de enfermería) no entienden eso, ellos creen que trayendo un pegoste bien bravo hacen bastante." CP_I_Bi_04
CUADRO 2. Características de los casos notificados con tuberculosis pulmonar en población indígena del Cauca, Colombia (2016-2017)

\begin{tabular}{|c|c|c|}
\hline \multirow[t]{2}{*}{ Características } & \multicolumn{2}{|c|}{ Población indígena } \\
\hline & $n=106$ & $\%$ \\
\hline \multicolumn{3}{|l|}{ Sexo } \\
\hline Masculino & 60 & 56,6 \\
\hline Femenino & 46 & 43,4 \\
\hline Edad al diagnóstico (años) ${ }^{\mathrm{a}}$ & 41,5 & $(24-61)$ \\
\hline$\leq 15$ & 10 & 9,4 \\
\hline $16-35$ & 33 & 31,1 \\
\hline $36-55$ & 24 & 22,7 \\
\hline$\geq 56$ & 39 & 36,8 \\
\hline \multicolumn{3}{|l|}{ Período de notificación } \\
\hline 2016 & 38 & 35,9 \\
\hline 2017 & 68 & 64,1 \\
\hline \multicolumn{3}{|l|}{ Municipio de procedencia del caso } \\
\hline Caldono & 14 & 13,2 \\
\hline Toribio & 13 & 12,3 \\
\hline Santander de Quilichao & 10 & 9,4 \\
\hline Timbiquí & 10 & 9,4 \\
\hline Páez & 8 & 7,5 \\
\hline Corinto & 7 & 6,6 \\
\hline La Vega & 7 & 6,6 \\
\hline Jambaló & 5 & 4,7 \\
\hline Silvia & 5 & 4,7 \\
\hline Caloto & 4 & 3,8 \\
\hline Inzá & 4 & 3,8 \\
\hline Piendamó & 4 & 3,8 \\
\hline Otros ${ }^{b}$ & 15 & 14,2 \\
\hline \multicolumn{3}{|l|}{ Pueblo indígena } \\
\hline Nasa & 75 & 70,8 \\
\hline Eperara Siapidara & 13 & 12,3 \\
\hline Yanacona & 9 & 8,5 \\
\hline Misak & 8 & 7,5 \\
\hline Inga & 1 & 0,9 \\
\hline \multicolumn{3}{|l|}{ Régimen de afiliación } \\
\hline Subsidiado & 101 & 95,3 \\
\hline Contributivo & 3 & 2,8 \\
\hline No asegurado & 2 & 1,9 \\
\hline Condición de ingreso al programa & & 90 \\
\hline Nuevo & 96 & 90,6 \\
\hline Reingreso tras recaída & 10 & 9,4 \\
\hline Coinfección con VIH & 4 & 3,8 \\
\hline PSF realizadas & 3 & 2,8 \\
\hline Farmacorresistencia identificada & 1 & 0,9 \\
\hline \multicolumn{3}{|l|}{ Condición de egreso del programa } \\
\hline Curado & 75 & 70,8 \\
\hline Tratamiento terminado & 24 & 22,7 \\
\hline Fallecido durante el tratamiento & 5 & 4,7 \\
\hline No evaluado & 1 & 0,9 \\
\hline Pérdida en el seguimiento & 1 & 0,9 \\
\hline
\end{tabular}


FIGURA 1. Cascada de atención para la tuberculosis pulmonar y sus explicaciones en la población indígena del Cauca, Colombia (2016-2017)

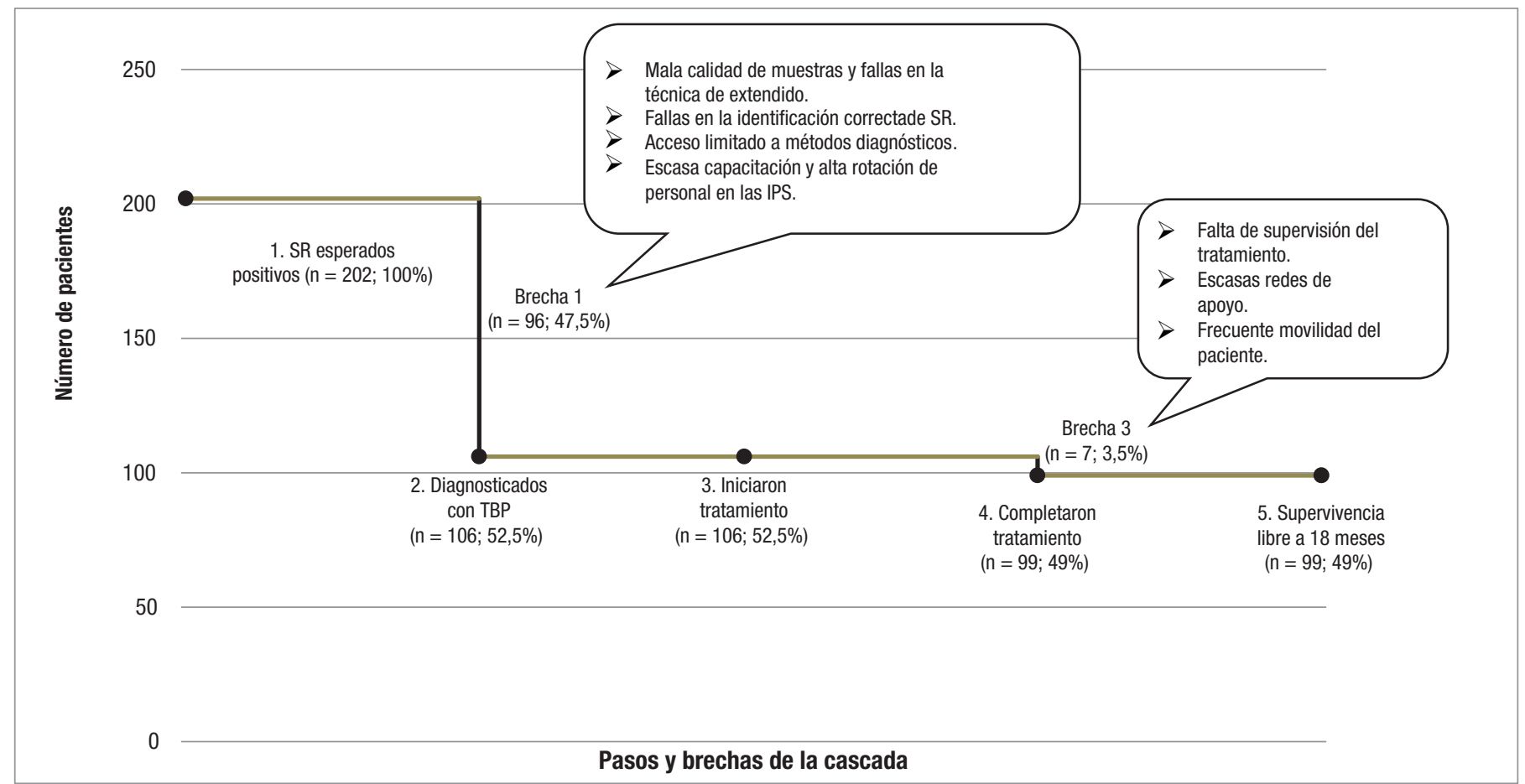

Fuente: elaboración propia con datos tomados del Departamento Administrativo Nacional de Estadística (DANE), base de datos del programa departamental de control de la tuberculosis, informes trimestrales

nacimientos y defunciones (RUAF) y hallazgos de la fase cualitativa.
SR, sintomático respiratorio; TBP, tuberculosis pulmonar; IPS, instituciones prestadoras de servicios de salud.

Fallas en la identificación correcta del SR. En los relatos se percibe que la presión de cumplir con una meta de captación ha llevado a que el personal de salud, en especial los auxiliares de enfermería que realizan la búsqueda activa, tomen muestras a personas que no cumplen la definición de SR. En ocasiones, para cumplir con la realización de las BK seriadas, se recurre a prácticas inadecuadas como la distribución en dos láminas de una misma muestra de esputo. De igual forma, las condiciones de transporte de muestras no garantizan su viabilidad.

"...es muy complicado que recojan las tres muestras unos (auxiliares de enfermería) nomás recogen dos, ...de la segunda nosotros sacamos dos para tener las tres." AP_I_Bi_04

“...que tengamos algo así como específico (para transportar las muestras) no, yo lo hecho en una bolsa y lo llevo para la casa o igual uno la lleva en la mano y ya." AP_I_Bi_07

Acceso limitado a métodos diagnósticos como cultivo y pruebas moleculares. Los entrevistados manifiestan que el análisis de las muestras de esputo se reduce a la BK, que es fácil y asequible dada la infraestructura de las IPS primarias. No se suelen utilizar los cultivos debido a que los laboratorios que realizan este procedimiento se encuentran en la ciudad capital. Caso similar ocurre con las pruebas moleculares. Por otra parte, los trámites administrativos para la realización de los exámenes de laboratorio en las aseguradoras y la oferta nula de laboratorios especializados en los territorios contribuyen a este problema, lo que aumenta la fragmentación de los servicios.
"Tendría que ir a su EPS (empresa promotora de salud o aseguradora), para que su EPS le gestione la salida del usuario hasta la ciudad donde le encuentren la cita." CP_E_Ai_01.

"Sí es baciloscopia pues sí lo que es de acá de primer nivel, rayos $X$ les toca en (municipio a 20 kilómetros) y PPD (prueba de tuberculina) en (municipio a 50 kilómetros)." CP_I_Bi_04

Escasa capacitación y alta rotación de personal al interior de las IPS. Las auxiliares de enfermería manifiestan que hay pocos procesos de capacitación continua en TB. Además, es frecuente la rotación de personal. Los profesionales de medicina y enfermería evitan laborar en IPS ubicadas en municipios de difícil acceso o con problemas de orden público. Por su parte, el personal técnico se renueva con una frecuencia que varía entre seis meses a dos años, por políticas internas de los cabildos, que administran las IPS indígenas.

"Capacitación de tuberculosis en sí no nos han dado, lo que hacemos es que leemos información de internet, por ejemplo." AP_I_Bi_09

\section{Brecha 3: explicaciones frente a los pacientes con fracaso, pérdida de seguimiento del tratamiento o muerte}

Barreras para la supervisión o tratamiento autoadministrado. Los coordinadores de programa refieren que, cuando 
CUADRO 3. Cálculos y limitaciones de la cascada de atención de la tuberculosis pulmonar en la población indígena del Cauca, Colombia (2016-2017)

\begin{tabular}{|c|c|}
\hline Pasos y brechas & Cálculo \\
\hline Paso 1 & $\begin{array}{l}\text { Supuesto 1. Proporción de mayores de } 15 \text { años de la proyección poblacional en el departamento del Cauca para } 2016=72,1 \% \\
\text { Supuesto 2. Proporción de mayores de } 15 \text { años de la proyección poblacional en el departamento del Cauca para } 2017=72,5 \% \\
\text { Supuesto 3. Proyección de la población indígenas en el departamento del Cauca para } 2016=344922 \text { indígenas } \\
\text { Supuesto 4. Proyección de la población indígenas en el departamento del Cauca para } 2017=287295 \text { indígenas } \\
\text { Supuesto 5. Mayores de } 15 \text { en población indígena para } 2016 \text { = supuesto } 3 \times \text { supuesto } 1 / 100=248668 \text { indígenas } \\
\text { Supuesto 6. Mayores de } 15 \text { en población indígena para } 2017=\text { supuesto } 4 \times \text { supuesto } 2 / 100=208288 \text { indígenas } \\
\text { Supuesto 7. } 50 \% \text { de mayores de } 15 \text { en población indígena para } 2016-2017=\text { supuesto } 5+\text { supuesto } 6 \times 0,5=228478 \\
\text { Supuesto 8. } 5 \% \text { de la mitad de los mayores de } 15 \text { en población indígena para } 2016-2017=\text { supuesto } 7 \times 0,05=11423 \\
\text { Supuesto 9. Total de SR esperados en la población indígena del Cauca en Colombia } 2016-2017=11423 \\
\text { Supuesto 10. Índice de positividad observado para la baciloscopia en Colombia durante } 2015=1,77 \% \\
\text { Total de SR esperados positivos en la población indígena del Cauca en Colombia } 2016-2017=\text { supuesto } 9 \times \text { supuesto } 10 / 100=202\end{array}$ \\
\hline Brecha 1 & Total de pacientes con TBP no diagnosticados = paso $1-$ paso $2=96$ \\
\hline Paso 2 & NT de PIN con TBP durante 2016-2017 = 106 \\
\hline Paso 4 & NT de PIN con TBP durante 2016-2017 que lograron la curación o la finalización del tratamiento = 99 \\
\hline Brecha 4 & $\begin{array}{l}\text { Supuesto. NT de PIN con TBP durante 2016-2017 que iniciaron, luego finalizaron tratamiento y fallecieron durante los primeros } 18 \text { meses posterior al } \\
\text { egreso del programa }=0\end{array}$ \\
\hline Paso 5 & $\begin{array}{l}\text { NT de PIN con TBP durante 2016-2017 que iniciaron, luego finalizaron tratamiento y permanecen vivos durante los primeros } 18 \text { meses posterior al egreso } \\
\text { del programa = paso } 4 \text { - brecha } 4=99 \text { casos }\end{array}$ \\
\hline
\end{tabular}

Fuente: elaboración propia.

NT, número total; SR: sintomáticos respiratorios; PIN, pacientes indígenas notificados; BDPD: base de datos del programa departamental, TBP, tuberculosis pulmonar

se diagnostica una persona que vive en una zona rural se dificulta la supervisión del tratamiento. Al respecto, se opta por capacitar a algún líder comunitario, familiar o incluso al mismo paciente para la administración.

"Le dimos los tratamientos, aunque era un riesgo grandisimo porque le dimos 6 meses así, hicimos un acuerdo y creo que él (usuario) no se los tomó, por eso volvió y salió positivo... El tratamiento estrictamente supervisado, el costo es muy alto, porque asignar al auxiliar que quede más cerca, pero todos los días ir hasta allá implicaba dos horas de camino, le toca caminar todos los días entonces es un agotamiento para el auxiliar." CP_E_Ai_09

Escasas redes de apoyo. La mayoría de los actores clave coinciden en que las personas con TB que viven solas o no cuentan con apoyo de familia o amigos son más vulnerables y pueden perder la motivación para continuar su proceso de recuperación.

“...el anterior año fue un poco complicado porque ni el paciente quería y la familia era un poco despegada de él, entonces la familia no vivía con él. Él vivía solo, pero vivía como a tres casas de la familia y ellos no iban al hospital, fue una auxiliar del hospital que vivía cerca por la zona que se encargaba de estarle llevando la medicación todos los dias." CP_E_Bi_02
Frecuente movilidad del paciente. Otra explicación es la movilidad frecuente por diferentes razones para la búsqueda de empleo, que dificulta el seguimiento por parte del personal de salud. Para tratar de contrarrestarlo, los cabildos suelen apoyar a sus comuneros enfermos con recursos económicos para los desplazamientos a los controles durante el tratamiento o con donaciones en especie como granos y abarrotes.

"...se nos pierden (los usuarios) y no los encontramos, se nos van para otro lado y nosotros andamos buscándolos" CP_E_Ai_09

"...bueno en realidad un paciente difícil porque estuvo como unas dos semanas, volvió y se fue, creo que fue para el Valle (otro departamento), allá recibió tratamiento otra vez, volvió ahorita hace como días y otra vez esta acá, entonces un poco difícil." CP_E_Bi_02

\section{DISCUSIÓN}

Este es el primer estudio en Colombia que analiza la cascada de atención de la TB en población indígena. Lo anterior cobra relevancia para la toma de decisiones a nivel local y aporta evidencia sobre este tema. Los hallazgos en la evaluación de la cascada mostraron una brecha de $47,5 \%$ entre los casos esperados positivos a la BK y los diagnosticados. Las explicaciones 
posibles fueron fallas en la calidad de las muestras, la técnica de extendido y la identificación de SR, el acceso limitado a métodos diagnósticos como el cultivo y las pruebas moleculares, la capacitación escasa y la rotación alta de personal en las IPS.

La mayoría de los estudios sobre la cascada de atención de la TB se han concentrado en grandes poblaciones, sin tener en cuenta un enfoque étnico. La cascada de atención de la TB realizada en la población general en India culminó en $43 \%$ y se especificaba, como último paso, la supervivencia libre de recurrencia a un año $(15,16)$. Por otro lado, en Sudáfrica la cascada de atención de la TB finalizó en 53\%, y se tomó como último paso el tratamiento exitoso (24). Estos hallazgos son similares a los encontrados en el presente estudio.

Las brechas de la cascada varían según la población estudiada. En India, Kenia y Uganda, la falta de diagnóstico de TB ha sido identificada como la principal brecha en la cascada de atención $(15,16,25)$. Estos hallazgos son consistentes con el presente estudio. Por otra parte, en Sudáfrica se identificó la falta de tratamiento exitoso como brecha importante en la cascada de atención (24), contrario a lo reportado en el presente estudio, donde la brecha fue mínima y las explicaciones encontradas no parecieron impactar en el resultado del indicador. Estas diferencias en los hallazgos podrían explicarse por los factores contextuales, el uso de diferentes pruebas diagnósticas y las diferencias en estimaciones en los pasos de la cascada.

Un estudio realizado en médicos indígenas en la India informó que $62 \%$ de ellos identificaban entre dos a cinco casos sospechosos de TB al mes. Sin embargo, solo $23 \%$ prescribió BK para diagnosticar la enfermedad (26). Desde un enfoque cualitativo, en Perú se informó la escasa disponibilidad de métodos diagnósticos como uno de los principales problemas en la comunidad indígena Ashaninka. No todos los centros de salud en esta comunidad tenían la capacidad de recolectar y transportar las muestras al laboratorio de referencia de manera oportuna (27). En otro estudio se encontró que más de $25 \%$ de las BK en indígenas correspondían a personas que lo solicitaban como requisito para ingreso laboral. Además, la exigencia de metas mensuales de cumplimiento al personal técnico hizo que los esfuerzos se enfocaran en lograr esos objetivos y se tomaron muestras a personas asintomáticas (28). Lo anterior es consistente con el hallazgo de fallas en la correcta identificación de SR en comunidades indígenas.

En comunidades indígenas de Colombia se identificó que una dificultad para el diagnóstico y tratamiento adecuado fue la escasez de vías de comunicación y dificultades en el desplazamiento hacia las ciudades donde se ubican las IPS, debido a la falta de un sistema de transporte público eficiente $(5,29)$. De igual manera, los profesionales de la salud que laboran en zonas rurales del Perú han reconocido el acceso limitado a las herramientas diagnósticas para la TB en los centros de atención (30). Lo anterior, junto con nuestros hallazgos confirma las limitaciones de acceso a métodos diagnóstico de TB por la centralización de estos en las ciudades.

Un estudio de la cascada de atención de la TB desde la perspectiva de los trabajadores de la salud en un entorno hospitalario indicó que la escasez de personal de enfermería tiene consecuencias negativas en la calidad de la atención (31). Lo anterior permite reflexionar que estas dificultades de los trabajadores de la salud son más marcadas en contextos de atención primaria de difícil acceso como en la presente investigación. En México, los trabajadores de salud comunitarios indígenas abordan las necesidades de salud de sus comunidades con una comprensión tanto alopática como tradicional de la medicina. Ellos garantizan un control integral de la TB en sus comunidades, las cuales se ven afectadas por una carga alta de enfermedad, dificultades de accesibilidad geográfica y de orden público, e inadecuada infraestructura de atención médica (32). Lo anterior, junto a los hallazgos del presente estudio, sustenta la necesidad de incorporar y capacitar trabajadores de la salud indígenas en las IPS de manera sostenida en el tiempo. Esta estrategia ya ha sido estudiada con comunidades indígenas en Brasil donde, a través de la incorporación de agentes indígenas de salud en el sistema sanitario estatal, se facilita el acceso a los recursos biomédicos de salud, al tiempo que se reconoce la importancia de las prácticas de la medicina tradicional y otras como la autoatención (33).

Las brechas identificadas también podrían ser un reflejo de la falta de adecuación cultural de las políticas de control de la TB. En países como Australia, se ha identificado una escasa visibilidad de los pueblos indígenas en las políticas públicas y programas de control de la TB (34). Por lo anterior, se hace necesaria la elaboración conjunta de alternativas de control efectivas que cuenten con la participación de las comunidades indígenas, con base en los procesos culturales y sociales propios de cada pueblo y minimizando el despliegue vertical de intervenciones programáticas.

La cascada de la atención tiene dos beneficios potenciales: como un enfoque para cuantificar los resultados de la TB y como un marco conceptual para examinar la calidad de los servicios de salud en varias etapas de la atención. Las cascadas de atención de la TB se deben realizar en poblaciones de alto riesgo en países con una carga alta de la enfermedad (16). Estos contextos epidemiológicos se abordan en esta investigación y no han sido reportados en la literatura actual. Esta investigación aborda la calidad de programa de control de TB en población indígena del Cauca en Colombia desde un enfoque de investigación mixta, lo que permite un análisis del contexto (35).

El estudio planteó algunas limitaciones en la construcción de la cascada. Como se desconocía la prevalencia de la enfermedad en población indígena en Colombia, no fue posible la estimación de intervalos de confianza de $95 \%$, tal como lo proponen Subbaraman et al. $(15,16)$. Por lo anterior, se calculó el total de SR esperados positivos. Para esto, se tomó como referencia los lineamientos del programa, específicamente lo relacionado con la programación de SR y el índice de positividad de la BK observado en Colombia (18). Además, la estimación de SR esperados positivos se ajustó con base en la proyección de la población indígena del departamento según el último censo poblacional. A pesar de esto, se reconocen limitaciones en los datos de fuentes secundarias, debido a que el programa de control de TB no discrimina por pueblo indígena los SR.

En conclusión, los hallazgos que se muestran aquí revelan una brecha importante en la detección de TB, explicada en gran medida por la mala calidad de las muestras y fallas en la técnica del extendido, fallas en la correcta identificación del SR, acceso limitado a métodos diagnósticos como cultivo y pruebas moleculares, y escasa capacitación y alta rotación de personal al interior de las IPS. No se encontraron brechas entre los pacientes diagnosticados y tratados, y la brecha de finalización del tratamiento fue mínima; por lo que se hace necesario profundizar en futuras investigaciones que expliquen esta situación.

Para el mejoramiento del programa de control de TB en la población indígena se recomienda: i) fortalecer los procesos 
de capacitación continua al personal de salud mediante el desarrollo de contenidos estandarizados a través del uso de herramientas como las tecnologías de la información y comunicación, ii) explorar la implementación de pruebas rápidas de diagnóstico, iii) considerar la contratación de auxiliares de enfermería indígenas para la gestión del programa, y iv) involucrar a la comunidad indígena en la educación en prevención y control de TB en el contexto de diálogo de saberes para un abordaje más holístico.

Contribución de los autores. JEPP e IRM concibieron el concepto y el diseño, analizaron einterpretaron los datos y redactaron el manuscrito. KYTH contribuyó al concepto y diseño, apoyó la logística del trabajo de campo, adquisición de fuentes secundarias, y revisó críticamente el manuscrito. PTP y FP contribuyeron al concepto y diseño y revisaron críticamente el manuscrito. BLGS realizó las entrevistas semiestructuradas, analizó la información cualitativa y revisó críticamente el manuscrito. Todos los autores leyeron y aprobaron el manuscrito final.

\section{Conflicto de intereses. Ninguno declarado por los autores.}

Declaración. Las opiniones expresadas en este manuscrito son únicamente responsabilidad de los autores y no reflejan necesariamente los de la Revista Panamericana de Salud Pública o la Organización Panamericana de la Salud.

\section{REFERENCIAS}

1. World Health Organization. Global tuberculosis report 2019 [Internet]. Geneva; 2019 [citado 2019 Dic 26]. Disponible en: https:// apps.who.int/iris/bitstream/handle/10665/329368/978924156571 4-eng.pdf?ua $=1$

2. Cormier M, Schwartzman K, N'Diaye DS, Boone CE, Dos Santos AM, Gaspar J, et al. Proximate determinants of tuberculosis in Indigenous peoples worldwide: a systematic review. Lancet Glob Health. 2019;7(1):e68-80.

3. Tollefson D, Bloss E, Fanning A, Redd JT, Barker K, McCray E. Burden of tuberculosis in indigenous peoples globally: a systematic review. Int J Tuberc Lung Dis. 2013;17(9):1139-50.

4. Tuberculosis in the Americas, 2018 [Internet]. Washington, D.C.: PAHO; 2018 [citado 2018 Oct 21]. Disponible en: http://iris.paho. $\mathrm{org} / \mathrm{xmlui} /$ handle $/ 123456789 / 49510$ ?show $=$ full

5. Hernandez Sarmiento JM, Davila Osorio VL, Martinez Sanchez LM, Restrepo Serna L, Grajales Ospina DC, Toro Montoya AE, et al. Tuberculosis in indigenous communities of Antioquia, Colombia: epidemiology and beliefs. J Immigr Minor Health. 2013 Feb;15(1):10-6.

6. Bergonzoli G, Castellanos LG, Rodriguez R, Garcia LM. Determinants of tuberculosis in countries of Latin America and the Caribbean. Rev Panam Salud Publica. 2016;39(2):101-5.

7. Instituto Nacional de Salud. Informe del evento tuberculosis, Colombia, 2017 [Internet]. Instituto Nacional de Salud. Bogotá: INS; 2018 [citado 2018 Sep 28]. Disponible en: https:/ / www.ins.gov.co/ buscador-eventos/Informesdeevento/Tuberculosis 2017.pdf

8. Gobernación del Cauca. Registros del programa departamental de tuberculosis. Popayán; 2017.

9. Ministerio de Salud y Protección Social. Plan estratégico hacia el fin de la tuberculosis, 2016-2025 [Internet]. Colombia; 2017 [citado 2018 Sep 28]. Disponible en: https://www.minsalud.gov.co/sites/rid/ Lists / BibliotecaDigital/RIDE/INEC/INTOR/Plan-estrategicofin-tuberculosis-colombia-2016-2025.pdf

10. Perlman DC, Jordan AE, Nash D. Conceptualizing Care Continua: Lessons from HIV, Hepatitis C Virus, Tuberculosis and Implications for the Development of Improved Care and Prevention Continua. Front public Health. 2016;4:296.

11. World Health Organization. Implementation research toolkit [Internet]. WHO, editor. Geneva: WHO; 2014 [citado 2018 Oct 26]. Disponibleen:https://apps.who.int/iris/bitstream/handle/10665/ 110523/9789241506960_Workbook_eng.pdf? sequence $=3 \&$ is Allowed $=\mathrm{y}$

12. Creswell J, Plano V. Designing and conducting mixed methods research. 2nd ed. SAGE, editor. Washington DC: SAGE; 2011.

13. Teddlie C, Yu F. Mixed methods sampling: A typology with examples. J Mix Methods Res. 2007;1(1):77-100.

14. Ulin P, Robinson E, Tolley E. Investigación aplicada en salud pública: métodos cualitativos. Washington DC: OPS; 2006.

15. Subbaraman R, Nathavitharana RR, Satyanarayana $S$, Pai M, Thomas BE, Chadha VK, et al. The tuberculosis cascade of care in India's public sector: A systematic review and meta-analysis. PLoS Med. 2016;13(10):e1002149.
16. Subbaraman R, Nathavitharana RR, Mayer KH, Satyanarayana $\mathrm{S}$, Chadha VK, Arinaminpathy N, et al. Constructing care cascades for active tuberculosis: A strategy for program monitoring and identifying gaps in quality of care. PLOS Med. 2019;16(2):e1002754.

17. Del Portillo-Mustieles EC, Laniado-Laborín R. Active case finding of pulmonary tuberculosis through screening of respiratory symptomatics using sputum microscopy: is it time to change the paradigm? Tuberc Res Treat. 2013;2013:312824.

18. Ministerio de Salud y Protección Social. Circular externa No. 000055 de 2016 [Internet]. Colombia; 2016. Disponible en: https://www. minsalud.gov.co/sites/rid/Lists/BibliotecaDigital/RIDE/DE/ DIJ/circular-externa-55-de-2016.pdf

19. Daza Arana JE, Cubides Munévar AM, Lozada Ramos H. Prevalencia de sintomáticos respiratorios y factores relacionados en dos territorios vulnerables de Santiago de Cali. Hacia la Promoción la Salud. 2016;21:63-76.

20. World Health Organization. Definitions and reporting framework for tuberculosis-2013 revision [Internet]. Geneva: WHO 2013 [citado 2019 Dec 26]. Disponible en: https://apps.who. int/iris/bitstream/handle/10665/79199/9789241505345_eng. pdf? sequence $=1 \&$ isAllowed $=y$

21. Edwards R, Holland J. What is qualitative interviewing? London: Bloomsbury Publishing; 2013.

22. Fereday J, Muir-cochrane E. Demonstrating Rigor Using Thematic Analysis : A Hybrid Approach of Inductive and Deductive Coding and Theme Development. Int J Qual methods. 2006;5(1):80-92.

23. Hernández R, Fernández C, Baptista P. Metodología de la investigación. 5th ed. México: McGrawHill; 2010.

24. Naidoo P, Theron G, Rangaka MX, Chihota VN, Vaughan L, Brey ZO, et al. The South African tuberculosis care cascade: Estimated losses and methodological challenges. J Infect Dis. 2017;216(suppl_7):S702-13.

25. Mwangwa F, Chamie G, Kwarisiima D, Ayieko J, Owaraganise A, Ruel TD, et al. Gaps in the Child Tuberculosis Care Cascade in 32 Rural Communities in Uganda and Kenya. J Clin Tuberc other Mycobact Dis. 2017;9:24-9.

26. Anandhi CL, Nagaraj VK, Kumar R. Knowledge and practice pattern of non-allopathic indigenous medical practitioners regarding tuberculosis in a rural area of India. Int J Tuberc Lung Dis. 2002;6(6):553-5.

27. Gianella C, Ugarte-Gil C, Caro G, Aylas R, Castro C, Lema C. TB in vulnerable populations: The case of an indigenous community in the Peruvian Amazon. Health Hum Rights. 2016;18(1):55-68.

28. Gianella C, Pesantes MA, Ugarte-Gil C, Moore DAJ, Lema C. Vulnerable populations and the right to health: lessons from the Peruvian Amazon around tuberculosis control. Int J Equity Health. 2019;18(1):28.

29. Sánchez Lerma L, Carrillo Franco J, Ochoa Bernal Y, Pérez-Gutiérrez N. Aproximación a la situación de la Tuberculosis pulmonar en comunidades indígenas del municipio Puerto Gaitán, Meta, Colombia. Rev Habanera Ciencias Médicas. 2018;17(3):462-80. 
30. Anticona Huaynate CF, Pajuelo Travezano MJ, Correa M, Mayta Malpartida H, Oberhelman R, Murphy LL, et al. Diagnostics barriers and innovations in rural areas: insights from junior medical doctors on the frontlines of rural care in Peru. BMC Health Serv Res. 2015; 15:454.

31. Lisboa M, Fronteira I, Mason PH, Martins M do RO. National TB program shortages as potential factor for poor-quality TB care cascade: Healthcare workers' perspective from Beira, Mozambique. PLoS One. 2020;15(2):e0228927-e0228927.

32. Herce ME, Chapman JA, Castro A, Garcia-Salyano G, Khoshnood $\mathrm{K}$. A role for community health promoters in tuberculosis control in the state of Chiapas, Mexico. J Community Health. 2010;35(2):182-9.

33. Pontes AL, Rego S, Garnelo L, Pontes AL, Rego S, Garnelo L. La actuación de agentes indígenas de salud en la región de Alto Rio Negro, Brasil: relaciones entre autoatención y biomedicina. Desacatos [Internet]. 2018 [citado 2020 Sep 13];(58):84-103. Disponible en: http:/ / www.scielo.org. $\mathrm{mx} /$ scielo.php?script=sci_arttext\&pid= S1607-050X2018000300084\&lng=es\&nrm=iso\&tlng=es
34. Devlin S, MacLaren D, Massey PD, Widders R, Judd JA. The missing voices of Indigenous Australians in the social, cultural and historical experiences of tuberculosis: a systematic and integrative review. BMJ Glob Health. [Internet]. 2019;4(6):e001794. Disponible en: http://gh.bmj.com/content/4/6/e001794.abstract

35. McDowell A, Engel N, Daftary A. In the eye of the multiple beholders: Qualitative research perspectives on studying and encouraging quality of TB care in India. J Clin Tuberc Other Mycobact Dis. 2019;16:100111.

Manuscrito recibido el 18 de junio de 2020. Aceptado para su publicación, tras revisión, el 15 de setiembre de 2020. 


\section{Tuberculosis care cascade for the indigenous population in Colombia: an operational research study}

ABSTRACT Objective. Construct and evaluate the care cascade for pulmonary tuberculosis in the indigenous population of the department of Cauca (Colombia) and identify existing gaps.

Methods. Mixed-methods sequential explanatory design. In the first phase, the pulmonary tuberculosis care cascade for the indigenous population of Cauca was evaluated. Data were obtained from secondary sources and all cases diagnosed from 1 January 2016 to 31 December 2017 were included. In the second phase, semi-structured interviews were done with nine program coordinators and 11 nursing auxiliaries to explain identified gaps. Absolute and percentage values were estimated for each of the steps and gaps in the care cascade. Quantitative and qualitative results were triangulated.

Results. In 2016 and 2017, an estimated 202 patients with respiratory symptoms were expected to be positive and 106 cases of pulmonary tuberculosis were reported among the indigenous population of the department of Cauca. A gap of $47.5 \%$ was found for diagnosis, since only $52.5 \%$ of subjects were diagnosed in health services. This gap was explained by poor quality of samples and flawed smear techniques; flaws in correct identification of patients with respiratory symptoms; limited access to diagnostic methods, such as culture and molecular tests; and limited training and high turnover of personnel in health service provider institutions.

Conclusions. The tuberculosis control program should focus actions on bridging the gap in case detection in the indigenous population.

Keywords Tuberculosis; indigenous population; health services, indigenous; health care quality, access, and evaluation; Colombia.

\section{Cascata de atenção da tuberculose para os povos indígenas na Colômbia: pesquisa operacional}

RESUMO Objetivo. Elaborar e avaliar a cascata de atenção da tuberculose pulmonar na população indígena do Departamento de Cauca (Colômbia) e identificar as lacunas existentes.

Métodos. Metodologia mista com desenho sequencial explicativo. Na primeira fase, avaliamos a cascata de atenção da tuberculose pulmonar para a população indígena de Cauca. Os dados foram obtidos de fontes secundárias, incluindo todos os casos diagnosticados entre 1 de janeiro de 2016 e 31 de dezembro de 2017. $\mathrm{Na}$ segunda fase, realizamos entrevistas semiestruturadas com nove coordenadores do programa e 11 auxiliares de enfermagem para explicar as lacunas identificadas. Estimamos os valores absolutos e percentuais em cada uma das etapas e as lacunas na cascata de atenção. Os resultados quantitativos e qualitativos foram triangulados.

Resultados. Nos anos de 2016 e 2017, foi estimada a ocorrência de 202 casos com sintomas respiratórios com diagnóstico esperado positivo; no entanto, os serviços de saúde só diagnosticaram e notificaram 106 casos de tuberculose pulmonar na população indígena do Departamento de Cauca. Portanto, identificamos uma lacuna diagnóstica de 47,5\%, já que apenas 52,5\% dos casos receberam um diagnóstico nos serviços de saúde. As explicações para esta lacuna foram a má qualidade das amostras e falhas na técnica de esfregaço, falhas na identificação correta dos sintomas respiratórios, acesso limitado aos métodos de diagnóstico, tais como cultura e testes moleculares, bem como capacitação deficiente e alta rotatividade de pessoal nas instituições de saúde.

Conclusões. As ações do programa de controle da tuberculose devem se concentrar em reduzir a lacuna na detecção de casos na população indígena.

Palavras-chave Tuberculose; população indígena; serviços de saúde do indígena; qualidade, acesso e avaliação da assistência à saúde; Colômbia. 